\title{
Early gonadotropin-releasing hormone antagonist protocol in women with polycystic ovary syndrome: A preliminary randomized trial
}

\author{
Jae Jun Shin ${ }^{1,2}$, Kyung Eui Park', Young Min Choi ${ }^{1,3}$, Hye-Ok Kim ${ }^{4}$, Dong-Hee Choi ${ }^{5}$, Woo Sik Lee ${ }^{6}$, Jung-Hyun Cho ${ }^{6}$ \\ 'Department of Obstetrics and Gynecology, Seoul National University College of Medicine, Seoul; ${ }^{2}$ Her Yoo Jae Hospital, Goyang; ${ }^{3}$ Institute of \\ Reproductive Medicine and Population, Medical Research Center, Seoul National University College of Medicine, Seoul; ${ }^{4}$ Department of Obstetrics and \\ Gynecology, Cheil General Hospital and Women's Healthcare Center, Dankook University College of Medicine, Seoul; ${ }^{5}$ Department of Obstetrics and \\ Gynecology, CHA Bundang Fertility Center, CHA University School of Medicine, Seongnam; ${ }^{6}$ Department of Obstetrics and Gynecology, CHA Gangnam \\ Fertility Center, CHA University School of Medicine, Seoul, Korea
}

Objective: To prospectively evaluate the efficacy and safety of a fixed early gonadotropin-releasing hormone (GnRH) antagonist protocol compared to a conventional midfollicular GnRH antagonist protocol and a long GnRH agonist protocol for in vitro fertilization (IVF) in patients with polycystic ovary syndrome (PCOS).

Methods: Randomized patients in all three groups (early antagonist, $n=14$; conventional antagonist, $n=11$; long agonist, $n=11$ ) received 21 days of oral contraceptive pill treatment prior to stimulation. The $\mathrm{GnRH}$ antagonist was initiated on the 1st day of stimulation in the early antagonist group and on the 6th day in the conventional antagonist group. The GnRH agonist was initiated on the 18th day of the preceding cycle. The primary endpoint was the number of oocytes retrieved, and the secondary endpoints included the rate of moderate-to-severe ovarian hyperstimulation syndrome (OHSS) and the clinical pregnancy rate.

Results: The median total number of oocytes was similar among the three groups (early, 16; conventional, 12; agonist, 19; $p=0.111$ ). The early GnRH antagonist protocol showed statistically non-significant associations with a higher clinical pregnancy rate (early, $50.0 \%$; conventional, 11.1\%; agonist, 22.2\%; $p=0.180$ ) and lower incidence of moderate-to-severe OHSS (early, 7.7\%; conventional, 18.2\%; agonist, 27.3\%; $p=0.463$ ), especially among subjects at high risk for OHSS (early, $12.5 \%$; conventional, $40.0 \%$; agonist, $50.0 \% ; p=0.324$ ).

Conclusion: In PCOS patients undergoing IVF, early administration of a GnRH antagonist may possibly lead to benefits due to a reduced incidence of moderate-to-severe OHSS in high-risk subjects with a better clinical pregnancy rate per embryo transfer. Further studies with more subjects are required.

Keywords: Gonadotropin-releasing hormone antagonist; In vitro fertilization; Ovarian hyperstimulation syndrome; Polycystic ovary syndrome; Pregnancy

Received: Apr 13, 2018 · Revised: Jun 21, 2018 · Accepted: Jun 23, 2018 Corresponding author: Young Min Choi

Department of Obstetrics and Gynecology, Seoul National University College of Medicine, 101 Daehak-ro, Jongno-gu, Seoul 03080, Korea

Tel: +82-2-2072-2385 Fax: +82-2-762-3599 E-mail:ymchoi@snu.ac.kr

*This study was supported by a grant from Merck Ltd., Republic of Korea.

This is an Open Access article distributed under the terms of the Creative Commons Attribution Non-Commercial License (http://creativecommons.org/licenses/by-nc/4.0/) which permits unrestricted non-commercial use, distribution, and reproduction in any medium, provided the original work is properly cited.

\section{Introduction}

Polycystic ovary syndrome (PCOS), the most common endocrine disorder in women of reproductive age [1-3], is also the most common cause of anovulatory infertility [4]. As women with PCOS represent a group of high responders, multi-follicular growth is easily achieved through controlled ovarian stimulation (COS). However, the risk of ovarian hyperstimulation syndrome (OHSS) is markedly elevat- 
ed in these patients [5].

The use of a gonadotropin-releasing hormone (GnRH) antagonist has advantages over $\mathrm{GnRH}$ agonist protocols in terms of a lower requirement for gonadotropins, reduced length of treatment, and reduced incidence of moderate-to-severe OHSS [6,7]. The GnRH antagonist is generally started either on a fixed schedule (i.e., day 6 of stimulation), or on a flexible schedule (i.e., appearance of a follicle greater than 14-15 mm in diameter). Both conventional methods start $\mathrm{GnRH}$ antagonists a few days after gonadotropin has been administered. In contrast, earlier initiation of the $\mathrm{GnRH}$ antagonist has been proposed to reduce exposure to luteinizing hormone $(\mathrm{LH})$ and estradiol $\left(\mathrm{E}_{2}\right)$ in the early follicular phase during $\mathrm{COS}$, which negatively affects the probability of pregnancy [8]. Additionally, early administration of the $\mathrm{GnRH}$ antagonist may increase the oocyte yield by eliminating the asynchrony (size discrepancy) among growing follicles [9]. In two previous studies, the early antagonist protocol showed a similar number of oocytes retrieved, a similar pregnancy rate, and reduced OHSS rates compared to the long agonist protocol in $\mathrm{PCOS}$ patients $[10,11]$.

The purpose of this study was to investigate the efficacy and safety of administering a GnRH antagonist on the 1st day of COS compared to the conventional $\mathrm{GnRH}$ antagonist protocol and long agonist protocol through a randomized trial.

\section{Methods}

\section{Study design}

This was a multi-center randomized parallel-group trial comparing three different protocols that incorporated different modes of $\mathrm{GnRH}$ analogue administration. The study complied with the Declaration of Helsinki and was approved by the Institutional Review Board of Seoul National University Hospital (IRB No. H-1012-114-345), and each participating hospital. This study has been registered at ClinicalTrials.gov (No. NCT01402336). Patients were enrolled from the following four university-based or university-affiliated hospitals, which submitted case report forms without rule violations: Seoul National University Hospital, CHA Gangnam Medical Center, CHA Bundang Medical Center, and Cheil General Hospital and Women's Healthcare Center.

Eligible participants had PCOS as defined by the Rotterdam criteria $[12,13]$. All women therefore had at least two of the three following characteristics: (1) oligo- or anovulation, (2) clinical and/or biochemical signs of hyperandrogenism, and (3) polycystic ovaries with the exclusion of other possible etiologies. Further inclusion criteria included: age 20-40 years; presence of both ovaries without ovarian tumors; a normal uterine cavity as assessed through an ultrasound scan, hysterosalpingograms, or hysteroscopy; normal renal, liver and hematological indices; normal thyroid function; and normal prolactin levels. The exclusion criteria were an extrauterine pregnancy or abortion in the past 3 months; abnormal gynecological bleeding of undetermined origin; a congenital uterine anomaly; congenital adrenal hyperplasia; androgen-secreting tumors; Cushing syndrome; any contraindication for recombinant follicle-stimulating hormone (rFSH), GnRH analogue, or human chorionic gonadotropin (hCG) administration; and severe male factor infertility. Eligible subjects were identified prospectively from women who planned to start an in vitro fertilization (IVF) or intracytoplasmic sperm injection (ICSI) treatment cycle. Written informed consent was obtained from each woman who wished to participate. After confirming patient eligibility, a blood test was performed to obtain the baseline female reproductive hormone profile, and height and weight were measured. Patients were randomized 1:1:1 to the following three groups using a webbased system: the day 1 antagonist group, the day 6 antagonist group, or the long agonist group. The web-based system was provided by the Medical Research Collaborating Center of Seoul National University Hospital (SNUH; https://mrcc.snuh.org). Stratified randomization was used (stratification according to the institution, block randomization with a randomly selected block size). The investigator was blind to the randomization process, including the size of each block. Neither patients nor doctors were blinded to the treatment assigned. Any patients who withdrew their consent continued with their routine IVF treatment and were excluded from the analysis. All serious adverse events were submitted to the Clinical Trials Unit, Seoul National University Hospital, and the trial was evaluated periodically by an independent data and safety monitoring board.

\section{Controlled ovarian stimulation}

The schematic representation of each protocol is shown in Figure 1. All subjects received oral contraceptive pills (OCPs) for 21 days starting on menstrual day 5 in the cycle prior to the treatment cycle. An OCP containing ethinyl $E_{2}(0.03 \mathrm{mg})$ and cyproterone acetate $(2 \mathrm{mg})$ (Diane-35; Bayer AG, Leverkusen, Germany) was recommended, but other OCPs were permitted. A starting dose of $150 \mathrm{IU}$ of $\mathrm{rFSH}$ (300 IU, 450 IU, 900 IU; Gonal-F, Merck Serono, London, UK) was recommended, but alterations were allowed if necessary (Table 1). GnRH analogues were administered as described below.

\section{1) The early antagonist group}

Patients in this group started daily $\mathrm{rFSH}$ treatment on the 3rd day of withdrawal bleeding following OCP administration. A subcutaneous dose of $0.25 \mathrm{mg}$ of cetrorelix ( $0.25 \mathrm{mg}$; Cetrotide, Merck Serono) was also started on the same day and continued daily through the day of hCG administration, inclusively. 


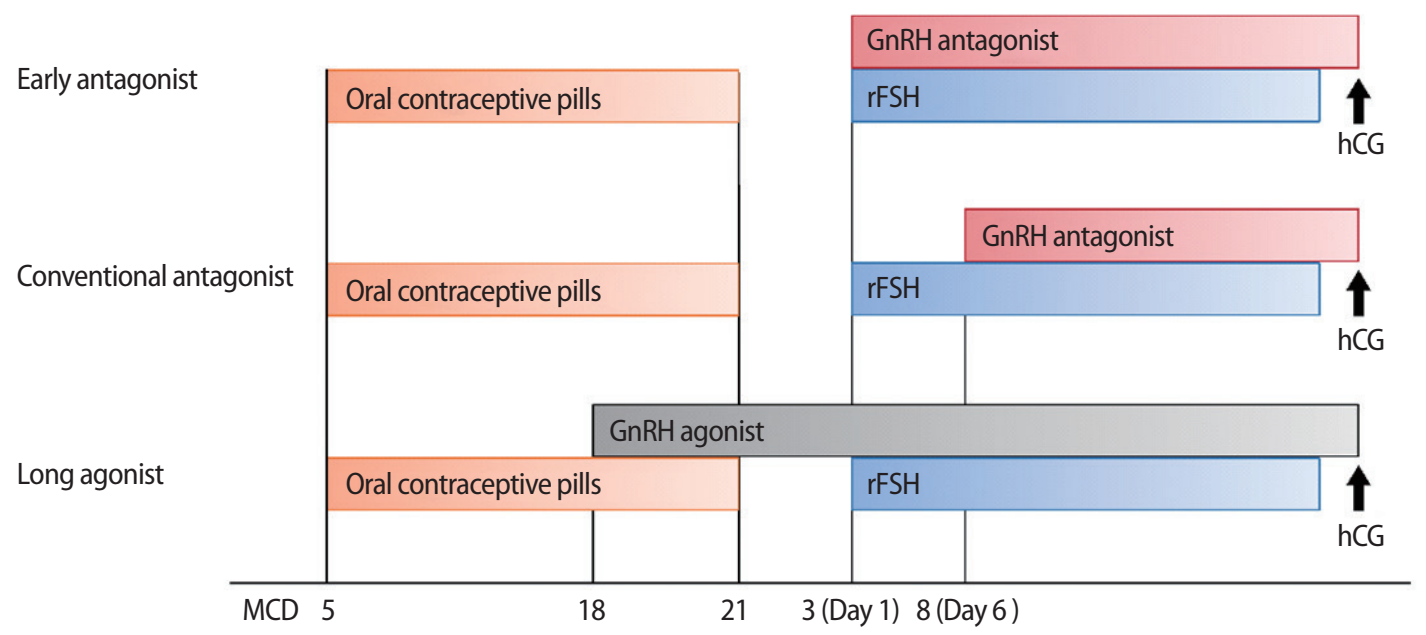

Figure 1. Schematic representation of each in vitro fertilization protocol. GnRH, gonadotropin-releasing hormone; rFSH, recombinant folliclestimulating hormone; $\mathrm{hCG}$, human chorionic gonadotropin; $\mathrm{MCD}$, menstrual cycle day.

Table 1. Baseline characteristics and hormonal profile of the study groups

\begin{tabular}{|c|c|c|c|c|}
\hline Variable & Early antagonist $(n=14)$ & Conventional antagonist $(n=11)$ & Long agonist $(n=11)$ & $p$-value \\
\hline Age (yr) & $32.5(29-39)$ & $33(25-37)$ & $33(31-40)$ & 0.234 \\
\hline $\mathrm{BMI}\left(\mathrm{kg} / \mathrm{m}^{2}\right)$ & $24.5(19.9-29.7)$ & $22.4(17.3-34.2)$ & $23.2(18.9-27.9)$ & 0.657 \\
\hline AFC & $40(25-69)$ & $36(18-100)$ & $31(20-70)$ & 0.647 \\
\hline \multicolumn{5}{|c|}{ Baseline hormonal profile } \\
\hline $\mathrm{AMH}(\mathrm{ng} / \mathrm{mL})$ & $9.8(4.7-15.7)$ & $9.8(4.1-19.8)$ & $6.8(4.2-15.6)$ & 0.061 \\
\hline $\mathrm{LH}(\mathrm{mlU} / \mathrm{mL})$ & $4.2(2.1-20.9)$ & $8.0(2.1-11.7)$ & $8.4(1.3-17.3)$ & 0.306 \\
\hline $\mathrm{FSH}(\mathrm{mlU} / \mathrm{mL})$ & $6.1(4.9-8.1)$ & $5.82(1.5-10.1)$ & $6.39(4.7-8.9)$ & 0.295 \\
\hline LH/FSH ratio & $0.7(0.4-3.0)$ & $1.0(0.5-3.7)$ & $1.2(0.3-2.7)$ & 0.329 \\
\hline $\mathrm{E}_{2}(\mathrm{pg} / \mathrm{mL})$ & $38(10-80)$ & $46(15-75)$ & $38(9-75)$ & 0.459 \\
\hline \multicolumn{5}{|l|}{ Patient in group } \\
\hline $\mathrm{AMH}>15(\mathrm{ng} / \mathrm{mL})$ & $1(9.1)$ & $3(27.3)$ & $1(9.1)$ & 0.613 \\
\hline $\mathrm{LH}>10(\mathrm{mlU} / \mathrm{mL})$ & $2(14.3)$ & $2(18.2)$ & $4(36.4)$ & 0.480 \\
\hline
\end{tabular}

Values are presented as median (range) or number (\%).

$\mathrm{BMI}$, body mass index; $\mathrm{AFC}$, antral follicle count; $\mathrm{AMH}$, anti-Müllerian hormone; $\mathrm{LH}$, luteinizing hormone; $\mathrm{FSH}$, follicle-stimulating hormone; $\mathrm{E}_{2}$, estradiol.

\section{2) The conventional antagonist group}

FSH treatment was initiated on the 3rd day of withdrawal bleeding and was continued as in the early antagonist group. A daily concomitant subcutaneous dose of $0.25 \mathrm{mg}$ of cetrorelix $(0.25 \mathrm{mg}$; Cetrotide, Merck Serono) was started on the 6th day of stimulation. Treatment with this GnRH antagonist continued daily through the day of hCG administration, inclusively.

\section{3) The long agonist group}

Patients received a subcutaneous dose of $0.1 \mathrm{mg}$ of triptorelin $(0.1$ mg; Decapeptyl, Ferring, Kiel, Germany) daily, starting on the 18th day of OCP administration (i.e., with 4 days of overlap). FSH treatment was initiated on the 3rd day of withdrawal bleeding and continued as in the early antagonist group and the conventional antagonist group. Starting from the 1st day of rFSH treatment, the dose of trip- torelin was reduced to $0.05 \mathrm{mg}$ daily and was continued through the day of $h C G$ administration, inclusively.

\section{Oocyte retrieval, fertilization, and transfer}

Recombinant hCG (250 $\mu$ g; Ovidrel, Merck Serono) or urinary hCG (10,000 IU; IVF-C, LG Life Sciences, Iksan, Korea) was administered when at least two follicles reached $17 \mathrm{~mm}$ in diameter. Oocyte retrieval was performed 36-38 hours following hCG administration, and fertilization was carried out using IVF, ICSI or half ICSI following the investigator's decision. For luteal support, daily vaginal progesterone (Crinone, Merck Serono) was started on the day of oocyte retrieval and was continued up to 8 weeks. When severe OHSS was anticipated, embryo transfer was waived, and all embryos were cryopreserved. Since many different criteria exist for performing cryopreservation of all embryos for OHSS prevention, and the purpose of 
this study was not to evaluate the OHSS prevention strategy, the decision was left to the clinicians. Embryo transfer was performed 2-5 days after oocyte retrieval, and a serum hCG test was performed 2 weeks after fertilization to confirm pregnancy. The gestational sac and fetal heartbeat were identified using transvaginal ultrasound in patients weekly thereafter.

\section{Assessment of embryo quality}

The morphological assessment of day 3 embryos was performed according to the following criteria: grade 1, blastomeres of equal size, no cytoplasmic fragments; grade 2, blastomeres of equal size, minor cytoplasmic fragments or blebs; grade 3, blastomeres of distinctly unequal size, few or no cytoplasmic fragments; grade 4, blastomeres of equal or unequal size, significant cytoplasmic fragmentation; grade 5 , few blastomeres of any size, severe or complete fragmentation. Good-quality embryos were defined as grade 1 or grade $2 \mathrm{em}$ bryos. Blastocysts were evaluated on day 5 using a scoring system based on the expansion state of the blastocyst and the consistency of the inner cell mass and trophectoderm cells. Good-quality embryos were defined as those with a score of $3 \mathrm{BB}$ or higher.

\section{Study endpoints}

The primary endpoint of this study was the number of oocytes retrieved. The secondary endpoints were the clinical pregnancy rate and the occurrence of moderate-to-severe OHSS. The classification by Golan and Weissman [14] was used to assess the severity of OHSS.

\section{Power analysis}

We set the baseline number of oocytes retrieved in the GnRH agonist long protocol for PCOS patients to be $13.2 \pm 5.8$ based on the study by Bahceci et al. [15] and the SNUH data (unpublished), and the detectable difference between groups at 4.9 , assuming an a level of 0.025 . The a level was halved since we compared the early antagonist group with two other groups. It was calculated that a sample size of 80 patients was required in each group to achieve a 0.80 power. Such a sample size (80 patients per group) is not easily achieved even in a multicenter study, when studying a small proportion of the population, such as PCOS patients who undergo IVF. The previous two studies on this topic reported 49 patients [10] and 78 patients [11] and we initiated this study because we thought we could provide useful clinical information, even with small number of subjects.

\section{Statistical analysis}

The results of this preliminary report are presented as median (range), and nonparametric tests were used for statistical analysis. Continuous variables, including the primary endpoint (number of oocytes retrieved) were compared using the Kruskal-Wallis test. The comparison of the frequency of features, including the secondary endpoints (the clinical pregnancy rate and the occurrence of moderate-to-severe OHSS) were performed using the chi-square test. All statistical analyses were performed using IBM SPSS ver. 22.0 (IBM Corp., Armonk, NY, USA). The a level of significance was set at 0.05 .

\section{Results}

\section{Patient enrollment and baseline characteristics}

Between February 2011 and December 2016, a total of 36 infertile women with PCOS were enrolled in this study, after the exclusion of seven women who discontinued participation after the initial agreement (Figure 2). One cycle in the early antagonist group was cancelled due to poor ovarian response, leaving a total of 35 cycles eligible for OHSS surveillance. No oocyte was retrieved in one patient in the conventional antagonist group. The cryopreservation of all embryos without embryo transfer was performed in a total of eight patients; seven of these procedures were performed to prevent OHSS, and one was due to hemoperitoneum. Embryo transfer was performed in the remaining 26 cycles. Age, body mass index, and baseline hormone levels were comparable in all three groups (Table 1). The characteristics of the cycles are shown in Table 2, and the IVF outcomes are shown in Table 3.

\section{Primary outcome}

The number of oocytes retrieved was greatest in the long agonist group $(n=19)$, followed by the early antagonist group $(n=16)$ and the conventional antagonist group $(n=12)$. However, the difference was not statistically significant $(p=0.111)$. The number of mature oocytes retrieved also followed a similar order, and likewise no statistically significant difference was observed among the early antagonist $(n=10.5)$, conventional antagonist $(n=8)$, and long agonist $(n=13)$ groups $(p=0.112)$.

\section{Secondary outcome}

The clinical pregnancy rate was greatest in the early antagonist group (50.0\%), followed by the long agonist group (22.2\%) and the conventional antagonist group (11.1\%), although this difference was not statistically significant $(p=0.180)$.

The incidence of moderate-to-severe OHSS was lowest in the early antagonist group (7.7\%), followed by the conventional antagonist group (18.2\%) and the long agonist group (27.3\%). However, this trend was not statistically significant $(p=0.463)$. The incidence of moderate-to-severe OHSS among patients at high risk for OHSS ( $\mathrm{E}_{2}$ concentration on hCG day $>2,000 \mathrm{pg} / \mathrm{mL}$ ) was lowest in the early antagonist group (12.5\%), in contrast to the conventional antagonist group (40\%) and the long agonist group (50.0\%), but this difference 


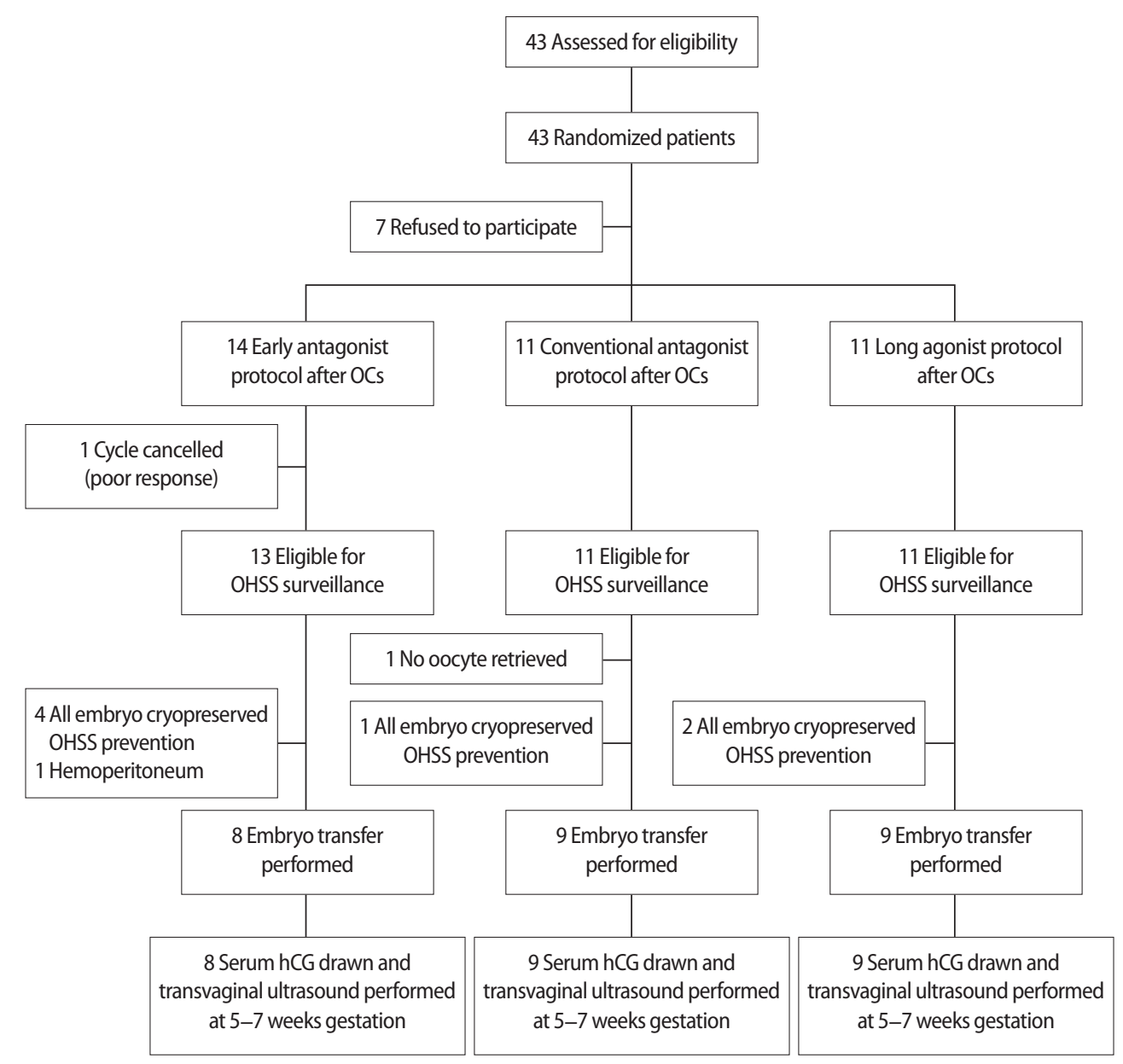

Figure 2. Flow diagram of the steps of the randomized trial. OCs, oral contraceptives; OHSS, ovarian hyperstimulation syndrome; hCG, human chorionic gonadotropin.

Table 2. Cycle characteristics in the study groups

\begin{tabular}{|c|c|c|c|c|}
\hline Variable & Early antagonist $(n=14)$ & Conventional antagonist $(n=11)$ & Long agonist $(n=11)$ & $p$-value \\
\hline Starting dose of gonadotropin (IU) & $150(150-225)$ & $150(150-225)$ & $225(150-300)$ & 0.074 \\
\hline Total dose of gonadotropin (IU) & $1,931(1,050-4,350)$ & $1,350(1,050-2,475)$ & $1,800(1,350-3,075)$ & 0.277 \\
\hline Duration of stimulation (day) & $10(7-13)$ & $9(7-12)$ & $10(7-17)$ & 0.483 \\
\hline Total ampules of antagonist & $11(8-14)$ & $4(2-7)$ & NA & $<0.001$ \\
\hline $\mathrm{E}_{2}$ concentration on day 6 of stimulation $(\mathrm{pg} / \mathrm{mL})$ & $1,542(107-3,914)$ & $648(108-3,635)$ & $348(90-906)$ & 0.634 \\
\hline $\mathrm{E}_{2}$ concentration on $\mathrm{hCG}$ day $(\mathrm{pg} / \mathrm{mL})$ & $3,795(614-9,020)$ & $969(109-9,006)$ & $2,216(1,067-3,217)$ & 0.109 \\
\hline Progesterone concentration on $\mathrm{hCG}$ day (ng/mL) & $0.9(0.6-2.2)$ & $0.5(0.4-0.7)$ & $0.5(0.2-0.8)$ & 0.621 \\
\hline Endometrial thickness on hCG day (mm) & $12(0.6-13.0)$ & $9.5(0.6-13.5)$ & $7(0.7-17.0)$ & 0.097 \\
\hline No. of follicles $\geq 11 \mathrm{~mm}$ on $\mathrm{hCG}$ day & $18(3-37)$ & $13.5(2-23)$ & $19(4-26)$ & 0.283 \\
\hline No. of follicles $\geq 14 \mathrm{~mm}$ on $\mathrm{hCG}$ day & $13(4-25)$ & $10(2-32)$ & $15(3-25)$ & 0.063 \\
\hline
\end{tabular}

Values are presented as median (range).

$\mathrm{NA}$, not applicable; $\mathrm{E}_{2}$, estradiol; $\mathrm{hCG}$, human chorionic gonadotropin.

was also not statistically significant $(p=0.324)$.

\section{Others}

The duration of stimulation was similar among the three groups. $A$ higher starting dose of FSH seemed to be preferred in the long ago- nist group, but there was no statistically significant difference among the three groups. The total dose of gonadotropin was greatest in the early antagonist group (median, 1,931 IU), followed by the long agonist group (median, 1,800 IU) and conventional antagonist group (median, 1,350 IU), but the difference was not statistically significant. 
Table 3. IVF outcomes of the study groups

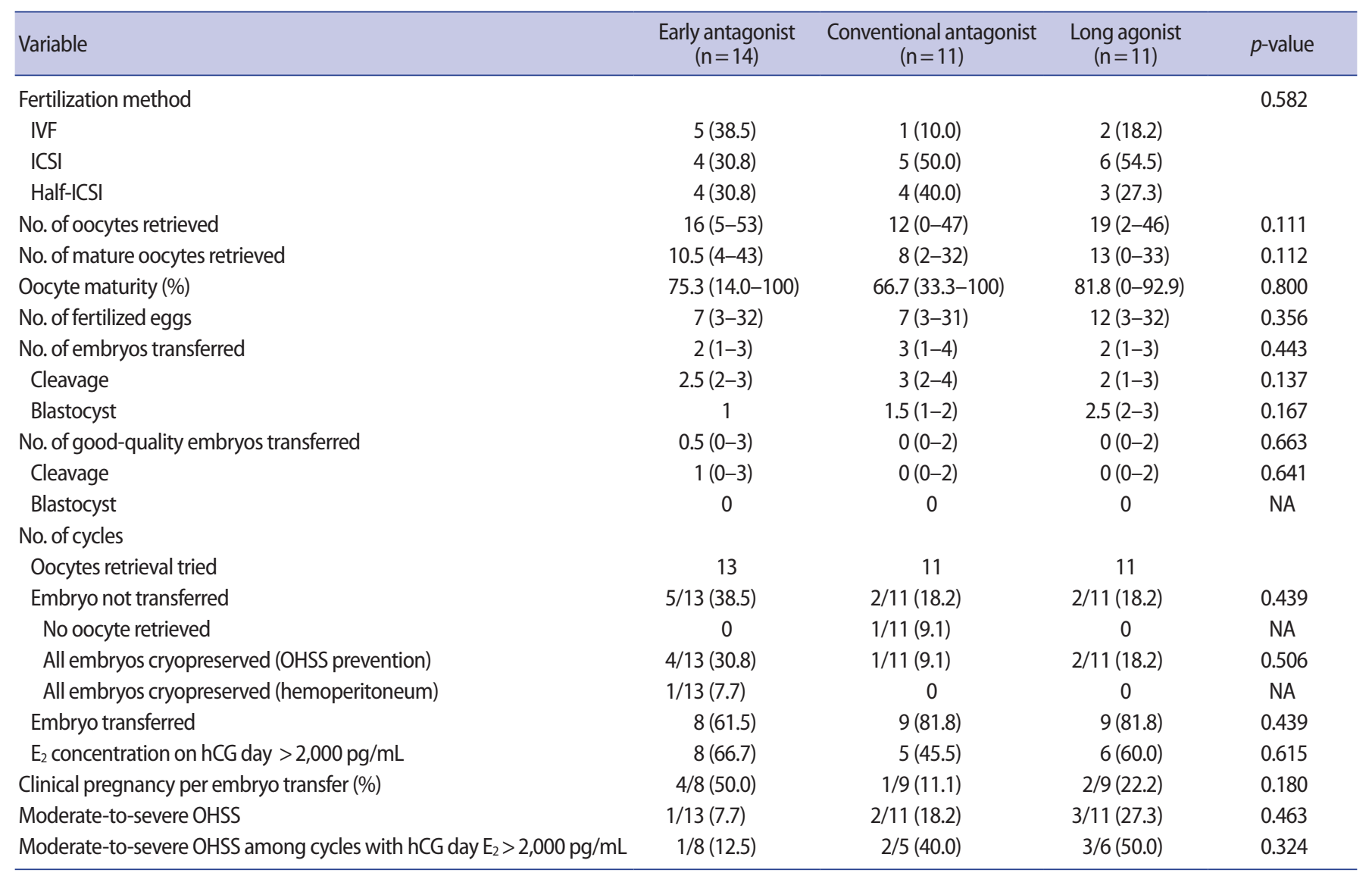

Values are presented as number (\%) or median (range).

IVF, in vitro fertilization; ICSI, intracytoplasmic sperm injection; NA, not applicable; OHSS, ovarian hyperstimulation syndrome; $E_{2}$, estradiol; hCG, human chorionic gonadotropin.

Although the $E_{2}$ concentration on day 6 seemed to be much higher in the early antagonist group than in the conventional antagonist group, the difference was not statistically significant. The $E_{2}$ concentration on the day of hCG administration was highest in the early antagonist group (median, 3,795 pg/mL), and was lowest in the conventional antagonist group (median, $969 \mathrm{pg} / \mathrm{mL}$ ), but the difference was not statistically significant. The number of large follicles on the hCG day was slightly higher in the early antagonist group than in the conventional antagonist group, but this difference did not reach statistical significance. There was no difference in oocyte maturity among the three groups $(p=0.800)$. The number of cycles that underwent embryo cryopreservation for OHSS prevention was greatest in the early antagonist group $(n=4)$. The reason for this may have been the high $E_{2}$ concentration on the day of hCG although the difference from other groups was not statistically significant. However, COS was not more intense in the patients with OHSS in the early antagonist group since the median total dose of gonadotropin was not significantly different between patients with OHSS $(2,006.25 \mathrm{IU})$ and patients without OHSS $(1,650 \mathrm{IU}, p=0.708)$.

\section{Discussion}

To our knowledge, this is the first randomized clinical trial to compare these three different protocols - namely, the early antagonist protocol, the conventional antagonist protocol, and the long agonist protocol-in PCOS patients. This prospective randomized study sought to determine whether there were differences in the number of oocytes retrieved, pregnancy outcomes, and the incidence of moderate-to-severe OHSS among three different COS protocols. We found no difference in the number of total oocytes and mature oocytes retrieved, the clinical pregnancy rate, or the incidence of moderate-to-severe OHSS among the three different protocols.

To prevent a premature LH surge during $\mathrm{COS}, \mathrm{GnRH}$ analogues are used to achieve pituitary suppression. GnRH agonists were the first to appear in clinical practice, and were commonly administered at the midluteal phase of the preceding menstrual period to avoid the initial flare effect (the $\mathrm{GnRH}$ agonist long protocol). The major disadvantages of the $\mathrm{GnRH}$ agonist long protocol are the long duration of administration, vasomotor symptoms associated with estrogen de- 
privation, the induction of unwanted ovarian cysts, and a high incidence of OHSS [16,17].

GnRH antagonists, introduced in the 2000s, could be administered during $\operatorname{COS}$. GnRH antagonists suppress the pituitary immediately by competing directly with endogenous GnRH. Compared to the GnRH agonist long protocol, the GnRH antagonist protocol has the advantages of a shorter duration of stimulation, reduced gonadotropin dose requirements, a lower cycle cancellation rate, lower peak $E_{2}$ levels, and a lower incidence of OHSS. The ongoing pregnancy rate and live birth rate have been found to be comparable to those obtained using long GnRH agonist protocols [7,18-20]. While administering a $\mathrm{GnRH}$ antagonist in the midfollicular phase has the above advantages, it was found that follicular development showed poor synchronization (i.e., greater size heterogeneity among growing follicles during COS) compared to the GnRH agonist long protocol, resulting in fewer follicles being visible on the day of $\mathrm{hCG}$ administration and fewer oocytes being retrieved. The absence of pituitary suppression during the first few days of COS may have caused the follicular asynchrony, and starting the $\mathrm{GnRH}$ antagonist earlier during COS may theoretically improve asynchrony among follicles, thereby increasing the oocyte yield and eliminating the potential detrimental effects of early follicular exposure to $\mathrm{LH}$ and $\mathrm{E}_{2}$ [8]. However, controversy emerged regarding whether earlier GnRH antagonist administration would increase the gonadotropin dose requirement, increase $E_{2}$ levels on the day of hCG, or have any effects on pregnancy outcomes and the incidence of OHSS $[8,9,11,21]$.

Several attempts have been made to introduce the early administration of GnRH antagonists during $\mathrm{COS}$ in women with PCOS. Hwang et al. [10] started a GnRH antagonist on the 3rd day following withdrawal bleeding after three cycles of OCP pretreatment. Gonadotropins were administered on the 4th day following withdrawal bleeding. Compared to the long agonist protocol, they found similar pregnancy rates ( $40 \%$ vs. $41.7 \%$, not significant) and similar OHSS rates ( $8.0 \%$ vs. $8.3 \%$, not significant), but the early antagonist protocol required less gonadotropin (21.6 \pm 6.3 vs. $25.9 \pm 7.2$ ampules of human menopausal gonadotropin, $p<0.05)$ and the peak $E_{2}$ level was lower on the day of $\mathrm{hCG}$ administration $(2,159 \pm 690 \mathrm{pg} / \mathrm{mL}$ vs. $2,633 \pm 775 \mathrm{pg} / \mathrm{mL}, p<0.05)$. Lainas et al. [11] also compared the early antagonist protocol with the long agonist protocol in $\mathrm{PCOS}$ patients and observed no difference in the number of oocytes retrieved ( 26.0 vs. $29.0, p=0.166), \mathrm{E}_{2}$ levels on the day of $\mathrm{hCG}$ administration $(2,333 \mathrm{pg} / \mathrm{mL}$ vs. $2,858 \mathrm{pg} / \mathrm{mL}, p=0.153)$, or clinical $(57.7 \%$ vs. $61.5 \%, p=0.808)$ and ongoing pregnancy rates $(46.2 \%$ vs. $48.1 \%, p=0.999$ ). However, the incidence of OHSS was significantly lower in the early antagonist group (11.54\%) than in the long agonist group $(38.46 \%, p=0.02)$.

Whether the early administration of $\mathrm{GnRH}$ antagonists improves follicular asynchrony has been an area of debate. Kolibianakis et al.
[21] reported no difference in the size distribution of follicles on the day of hCG administration between early and conventional antagonist groups in non-PCOS patients. Lainas et al. [11] reported that a similar number of oocytes was retrieved in the early antagonist protocol and in the long agonist protocol in PCOS patients. In contrast, a higher number of mature oocytes was retrieved in the early antagonist protocol than in the conventional antagonist protocol in nonPCOS patients [9]. Our results are similar to those of a study by Lainas et al. [11] and Kolibianakis et al. [21], and there was no difference in follicle size distribution or the number of total/mature oocytes retrieved.

The main strength of our study is that we compared the early antagonist protocol with two widely used protocols. The long agonist protocol mainly served as a control for the number of oocytes retrieved when there was excellent follicle synchronization. There was no difference in the follicle size distribution on the day of hCG administration or in the number of oocytes retrieved. In contrast, the conventional antagonist group served as controls to assess the effect of the additional 5 days of $\mathrm{GnRH}$ antagonist administration in terms of a possible increase in gonadotropin dose requirements as well as the primary and secondary outcomes. The total gonadotropin dose in the early antagonist group was higher than in the conventional antagonist group, but the difference was not statistically significant. Both the total number of oocytes and the number of mature oocytes were higher in the early antagonist group than in the conventional antagonist group, but these differences were likewise not statistically significant.

A major limitation of this study was the long recruitment phase. A possible explanation for this is that the candidates preferred a dedicated treatment plan and were reluctant to participate in a randomized trial during their fertility treatment. The small sample size was another limitation. There were differences in the number of oocytes retrieved, clinical pregnancy rates, and the incidence of moderate-tosevere OHSS, but statistical significance was not reached. Differences in those primary- and secondary outcome measures were not detected in our study with the possibility of type II error.

In conclusion, we compared three different ovarian stimulation protocols in PCOS patients. Early administration of a GnRH antagonist may possibly yield benefits due to a reduction in the incidence of moderate-to-severe OHSS in high-risk subjects with a similar number of oocytes retrieved, as well as a better clinical pregnancy rate per embryo transfer. To clarify these issues, further studies with more subjects are required.

\section{Conflict of interest}

This study was supported by a grant from Merck Ltd., Republic of 
Korea. The funder had no role in the design, collection, analysis or interpretation of this study. No other potential conflict of interest relevant to this article was reported.

\section{References}

1. Azziz R, Woods KS, Reyna R, Key TJ, Knochenhauer ES, Yildiz BO. The prevalence and features of the polycystic ovary syndrome in an unselected population. J Clin Endocrinol Metab 2004;89: 2745-9.

2. Diamanti-Kandarakis E, Kouli CR, Bergiele AT, Filandra FA, Tsianateli TC, Spina GG, et al. A survey of the polycystic ovary syndrome in the Greek island of Lesbos: hormonal and metabolic profile. J Clin Endocrinol Metab 1999;84:4006-11.

3. Knochenhauer ES, Key TJ, Kahsar-Miller M, Waggoner W, Boots LR, Azziz R. Prevalence of the polycystic ovary syndrome in unselected black and white women of the southeastern United States: a prospective study. J Clin Endocrinol Metab 1998;83:3078-82.

4. Hull MG. Epidemiology of infertility and polycystic ovarian disease: endocrinological and demographic studies. Gynecol Endocrinol 1987; 1:235-45.

5. Delvigne A, Demoulin A, Smitz J, Donnez J, Koninckx P, Dhont M, et al. The ovarian hyperstimulation syndrome in in-vitro fertilization: a Belgian multicentric study. I. Clinical and biological features. Hum Reprod 1993;8:1353-60.

6. Al-Inany $\mathrm{H}$, Aboulghar M. GnRH antagonist in assisted reproduction: a Cochrane review. Hum Reprod 2002;17:874-85.

7. Pundir J, Sunkara SK, El-Toukhy T, Khalaf Y. Meta-analysis of $\mathrm{GnRH}$ antagonist protocols: do they reduce the risk of OHSS in PCOS? Reprod Biomed Online 2012;24:6-22.

8. Kolibianakis EM, Albano C, Kahn J, Camus M, Tournaye H, Van Steirteghem AC, et al. Exposure to high levels of luteinizing hormone and estradiol in the early follicular phase of gonadotropin-releasing hormone antagonist cycles is associated with a reduced chance of pregnancy. Fertil Steril 2003;79:873-80.

9. Park CW, Hwang YI, Koo HS, Kang IS, Yang KM, Song IO. Early gonadotropin-releasing hormone antagonist start improves follicular synchronization and pregnancy outcome as compared to the conventional antagonist protocol. Clin Exp Reprod Med 2014;41:158-64.

10. Hwang JL, Seow KM, Lin YH, Huang LW, Hsieh BC, Tsai YL, et al. Ovarian stimulation by concomitant administration of cetrorelix acetate and HMG following Diane-35 pre-treatment for patients with polycystic ovary syndrome: a prospective randomized study. Hum Reprod 2004;19:1993-2000.

11. Lainas TG, Petsas GK, Zorzovilis IZ, Iliadis GS, Lainas GT, Cazlaris
$\mathrm{HE}$, et al. Initiation of GnRH antagonist on day 1 of stimulation as compared to the long agonist protocol in PCOS patients: a randomized controlled trial: effect on hormonal levels and follicular development. Hum Reprod 2007;22:1540-6.

12. Rotterdam ESHRE/ASRM-Sponsored PCOS Consensus Workshop Group. Revised 2003 consensus on diagnostic criteria and longterm health risks related to polycystic ovary syndrome. Fertil Steril 2004;81:19-25.

13. Rotterdam ESHRE/ASRM-Sponsored PCOS consensus workshop group. Revised 2003 consensus on diagnostic criteria and longterm health risks related to polycystic ovary syndrome (PCOS). Hum Reprod 2004;19:41-7.

14. Golan A, Weissman A. Symposium: update on prediction and management of OHSS. A modern classification of OHSS. Reprod Biomed Online 2009;19:28-32.

15. Bahceci M, Ulug U, Ben-Shlomo I, Erden HF, Akman MA. Use of a $\mathrm{GnRH}$ antagonist in controlled ovarian hyperstimulation for assisted conception in women with polycystic ovary disease: a randomized, prospective, pilot study. J Reprod Med 2005;50:84-90.

16. Asch RH, Li HP, Balmaceda JP, Weckstein LN, Stone SC. Severe ovarian hyperstimulation syndrome in assisted reproductive technology: definition of high risk groups. Hum Reprod 1991;6:1395-9.

17. Forman RG, Frydman R, Egan D, Ross C, Barlow DH. Severe ovarian hyperstimulation syndrome using agonists of gonadotropinreleasing hormone for in vitro fertilization: a European series and a proposal for prevention. Fertil Steril 1990;53:502-9.

18. Lainas TG, Sfontouris IA, Zorzovilis IZ, Petsas GK, Lainas GT, Alexopoulou $\mathrm{E}$, et al. Flexible $\mathrm{GnRH}$ antagonist protocol versus $\mathrm{GnRH}$ agonist long protocol in patients with polycystic ovary syndrome treated for IVF: a prospective randomised controlled trial (RCT). Hum Reprod 2010;25:683-9.

19. Al-Inany HG, Youssef MA, Ayeleke RO, Brown J, Lam WS, Broekmans FJ. Gonadotrophin-releasing hormone antagonists for assisted reproductive technology. Cochrane Database Syst Rev 2016;4:CD001750.

20. Griesinger G, Diedrich K, Tarlatzis BC, Kolibianakis EM. GnRH-antagonists in ovarian stimulation for IVF in patients with poor response to gonadotrophins, polycystic ovary syndrome, and risk of ovarian hyperstimulation: a meta-analysis. Reprod Biomed Online 2006;13:628-38.

21. Kolibianakis EM, Albano C, Camus M, Tournaye H, Van Steirteghem AC, Devroey P. Initiation of gonadotropin-releasing hormone antagonist on day 1 as compared to day 6 of stimulation: effect on hormonal levels and follicular development in in vitro fertilization cycles. J Clin Endocrinol Metab 2003;88:5632-7. 